\title{
A Case of Atrioventricular Nodal Reentrant Tachycardia With Atresia of the Coronary Sinus Ostium
}

\author{
Yuji OKuYAma, ${ }^{1 *} \mathrm{MD}$, Takafumi OKA,${ }^{1 *} \mathrm{MD}$, Hiroya Mizuno, ${ }^{1} \mathrm{MD}$, \\ Taku SAKAI, ${ }^{1}$ MD, Atsushi HIRAYAMA, ${ }^{1}$ MD, and Kazuhisa KodAMA, ${ }^{1}$ MD
}

\begin{abstract}
SUMMARY
We report a case of atrioventricular nodal reentrant tachycardia coexistent with atresia of the coronary sinus ostium. Radiofrequency current application between the supposed coronary sinus ostium and the tricuspid valve was effective at eliminating the tachycardia. A coronary venogram obtained by left coronary arteriography was useful for guiding the mapping catheter to the successful ablation site. (Int Heart J 2005; 46: 899-902)
\end{abstract}

Key words: Atrioventricular nodal reentry, Coronary sinus atresia, Kyphosis

$\mathrm{IN}_{\mathrm{N}}$ the era of radiofrequency catheter ablation of arrhythmias, interest in the coronary sinus is growing because its catheterization offers a landmark in guiding the procedure, particularly in those patients with supraventricular tachycardia. The incidences of coronary sinus abnormalities, such as coronary sinus diverticulum ${ }^{1)}$ and atresia of the coronary sinus ostium, ${ }^{2}$ in this subset of patients are not negligible (2.9\%). ${ }^{3)}$ We report a case of atrioventricular nodal reentrant tachycardia (AVNRT) associated with atresia of the coronary sinus ostium which was successfully eliminated by means of radiofrequency current applications.

\section{CASE REPORT}

A 58-year-old woman with recurrent palpitations was referred to our hospital for radiofrequency catheter ablation. A 12-lead ECG during sinus rhythm showed no delta wave. ECG during palpitations demonstrated a regular, narrow QRS tachycardia at a rate of 150 beats/min without a visible $\mathrm{P}$ wave in any leads. Physical examination revealed congenital kyphosis (Figure 1). After written informed consent was obtained from the patient, an electrophysiologic study was performed after withdrawal of antiarrhythmic drugs for five elimination halflives. Six-French and 5-French quadripolar catheters were introduced from the

From the ${ }^{1}$ Cardiovascular Division, Osaka Police Hospital, Osaka, Japan. The first two authors* contributed equally to this report.

Address for correspondence: Yuji Okuyama, MD, Cardiovascular Division, Osaka Police Hospital, 10-31 Kitayama-cho,

Tennoji-ku, Osaka 543-0035, Japan.

Received for publication March 22, 2005.

Revised and accepted June 10, 2005. 


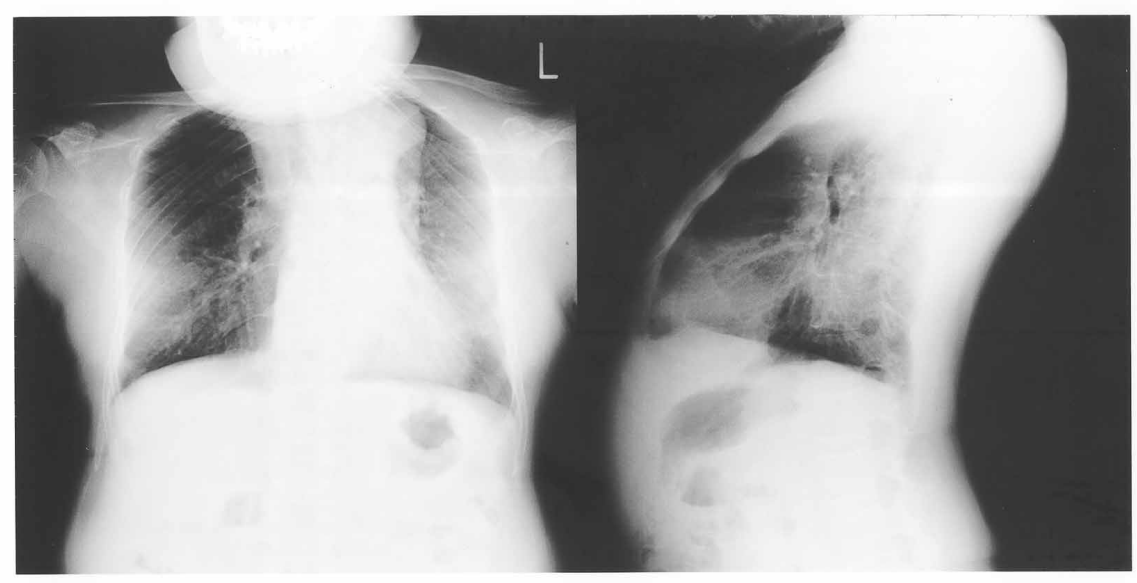

Figure 1. Chest radiographs demonstrating a severely malformed spine (kyphosis).

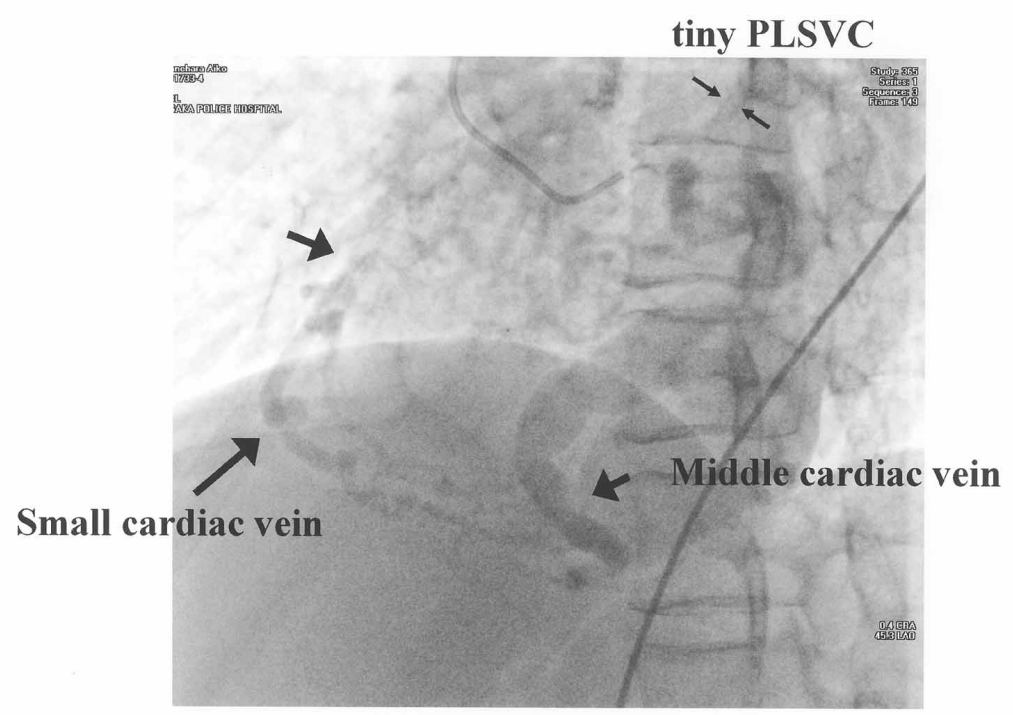

Figure 2. Left coronary angiography showing the atresia of the coronary sinus ostium with a dilated middle cardiac vein and a dilated small coronary vein drawing into the right atrium (arrow in left upper corner). A small tiny persistent left superior vena cava (PLSVC) was barely visible.

right femoral vein and placed in the high right atrium and near the His-bundle region, respectively. A 7-French decapolar catheter was introduced from the right antecubital vein and advanced to the area near the ostium of the coronary sinus (CS). However, we were unable to advance the catheter into the CS and then pro- 
ceeded to perform coronary angiography to obtain the venous phase of the left coronary angiogram. The CS was found to drain into the right atrium via an anomalous dilated vein running along the right coronary artery with the atresia of the ostium of the CS (Figure 2). As the patient did not have a persistent left superior vena cava large enough to cannulate an electrode catheter, we performed electrophysiological study without electrograms from the CS. A single atrial extrastimulation induced narrow QRS tachycardia with a sudden AH interval jump. During the tachycardia, the earliest atrial activation was recorded at the His bundle region. Right ventricular extrastimulation during the tachycardia could not reset the atrium, leading to the diagnosis of slow-fast type AVNRT. A 7French ablation catheter with a large-tip electrode was introduced from the right femoral vein to map the posteroseptal area of the right atrium, seeking the socalled slow pathway potential. Radiofrequency energy was delivered at the slow pathway potential recording site with the local atrioventricular ratio of about 0.2 at a temperature of $60^{\circ} \mathrm{C}$, resulting in nonsustained junctional rhythm with $1: 1$ retrograde atrial activation. After an application, AVNRT could no longer be induced with or without isoproterenol infusion ( $4 \mu \mathrm{g} / \mathrm{min})$.

\section{DISCUSSION}

An anomaly of atresia of the CS ostium was found incidentally in an electrophysiologic study in a patient with AVNRT (Figure 3). Slow pathway ablation at the usual site, that is, between the supposed coronary sinus ostium and the tricuspid valve, was effective at eliminating the tachycardia.

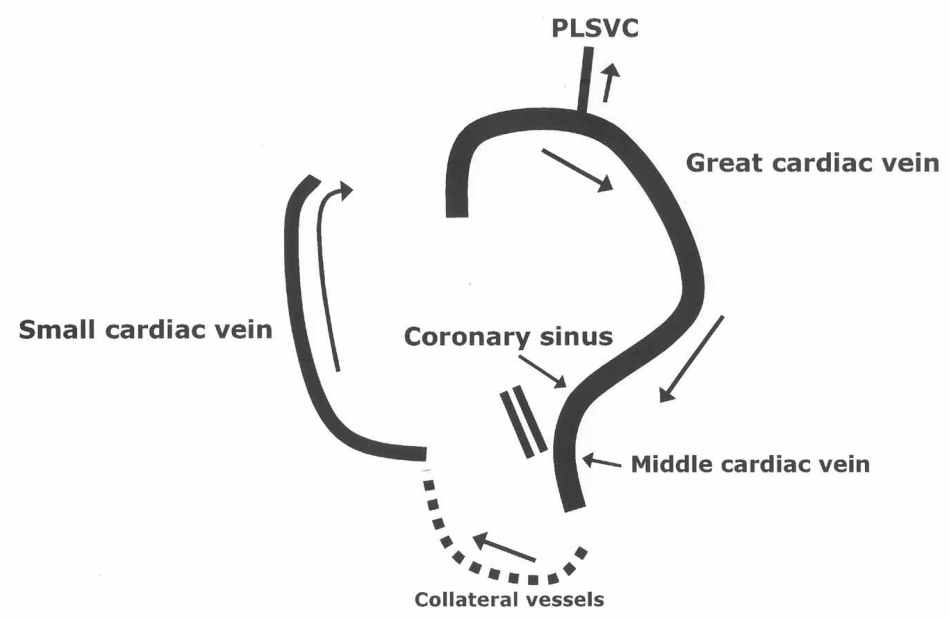

Figure 3. Schematic representation of coronary vein system. See Figure 2 for abbreviation. 
Atresia of the CS ostium is considered a rare cardiac venous anomaly. ${ }^{4}$ Coexistence of an accessory pathway with atresia of the CS ostium has been reported. ${ }^{2)}$ However, to the best of our knowledge, this is the first report of a patient with atresia of the CS ostium associated with AVNRT. It is possible that the coexistence of the coronary venous anomaly and the tachycardia occurred only by chance, however, there have been several reports showing a relation between the anatomical characteristics of the CS ostium and AVNRT. Doig, et al reported that the CS ostium in patients with AVNRT was larger and remained more dilated to at least $10 \mathrm{~mm}$ from the ostium than that in control. ${ }^{5)}$

In patients with a cardiac anomaly, the course of the input fibers into the AV nodal slow pathway has never been delineated. We placed the ablation catheter in the common area for slow pathway ablation, i.e., the posteroinferior region of Koch's triangle. The ratio of atrial/ventricular potentials at the successful ablation site was about 0.2 , suggesting a typical course of the AV nodal slow pathway fiber, that is near the tricuspid annulus.

In conclusion, catheter ablation using an RF current is feasible and safe in patients with slow-fast AVNRT associated with atresia of the CS ostium.

\section{REFERENCES}

1. Okishige K, Fisher JD, Goseki Y, et al. Radiofrequency catheter ablation for AV nodal reentrant tachycardia associated with persistent left superior vena cava. Pacing Clin Electrophysiol 1997; 20: 2213-8.

2. Takatsuki S, Mitamura H, Ieda M, Ogawa S. Accessory pathway associated with an anomalous coronary vein in a patient with Wolff-Parkinson-White syndrome. J Cardiovasc Electrophysiol 2001; 12: 1080-2.

3. Chiang CE, Chen SA, Yang CR, et al. Major coronary sinus abnormalities: identification of occurrence and significance in radiofrequency ablation of supraventricular tachycardia. Am Heart J 1994; 127: 1279-89.

4. Giebel J, Fanghanel J, Hauser S, Paul I. A case of a persistent left vena cava superior with atresia of the right atrial ostium of the coronary sinus. Ann Anat 2000; 182: 191-4.

5. Doig JC, Saito J, Harris L, Downar E. Coronary sinus morphology in patients with atrioventricular junctional reentry tachycardia and other supraventricular tachyarrhythmias. Circulation 1995; 92: 436-41. 